\title{
Fifteen-minute consultation: Time Out as an alternative to toxic debrief
}

\author{
Sian Cooper (D) ,' Mark Winton (D) ,' Joanna Farrington-Exley ${ }^{2}$
}

\begin{abstract}
${ }^{1}$ Paediatric Intensive Care Unit, Leeds Teaching Hospitals NHS Trust, Leeds, UK

${ }^{2}$ Paediatric Psychology Department, Leeds Teaching Hospitals NHS Trust, Leeds, UK
\end{abstract}

\section{Correspondence to}

Dr Sian Cooper, Paediatric Intensive Care Unit, Leeds Teaching Hospitals NHS Trust, Leeds LS2 9NS, UK; sian. cooper2@nhs.net

Received 25 April 2019 Revised 4 December 2019

Accepted 17 January 2020

Published Online First

11 February 2020

\section{Check for updates}

(C) Author(s) (or their employer(s)) 2020. No commercial re-use. See rights and permissions. Published by BMJ.

To cite: Cooper S, Winton M, Farrington-Exley J. Arch Dis Child Educ Pract Ed 2020;105:270-275

\begin{abstract}
Debriefing is well established in healthcare teams after acute events, with a focus on clinical learning, improving practice and performance; however, the term is perceived by psychologists as something quite different. This article describes the Time Out model as a standardised method of providing support to staff after events that may cause distress. In addition to exploring clinical issues, the model aims to promote peer support networks, educate staff regarding common reactions to traumatic events and signpost to other sources of support.
\end{abstract}

\section{INTRODUCTION}

Debriefing was developed for clinical learning in medical simulation and this practice has been applied in healthcare by clinical teams aiming to identify good practice, improve patient safety and team performance. ${ }^{1}$ Methods of debriefing have been described, ${ }^{2-5}$ but senior clinical staff often receive no training in how to deliver it effectively and there is little known about the impact on staff. ${ }^{6}$ A survey on the impact of child death on paediatricians in training in the UK found that feelings of guilt and attending a debrief may be associated with symptoms of acute stress reactions (ASR) or posttraumatic stress disorder (PTSD), ${ }^{7}$ while another study of intensive care staff found attending a debrief was associated with reduced risk of burnout. ${ }^{8}$

In contrast, the impact of psychological debriefing has been more extensively studied. Single session debriefing may increase the risk of PTSD and depression, ${ }^{9}$ and the National Institute for Health and Care Excellence in the UK advises against psychologically-focused debriefing for prevention of PTSD. ${ }^{10}$

Debrief is defined in box 1 and perhaps does not best describe what we hope to achieve in the healthcare setting. Staff in acute specialities are at risk of experiencing ASR, PTSD, moral distress and burnout (box 2), ${ }^{7} 81112$ and Health Education England emphasises the importance of supporting mental well-being in our staff. ${ }^{13}$ Cognitive behavioural therapy describes how thoughts, feelings, physical sensations and behaviours are all interconnected and each influences the other (figure 1). Time Out aims to support staff's understanding of events and their reactions (including their cognitions, emotions, physical responses and behaviours) in a way that is not harmful.

\section{HOW TO DEBRIEF WITHOUT CAUSING HARM?}

This is a crucial question. Enforced debriefs can interfere with natural coping mechanisms, ${ }^{14}$ and a single session without follow-up can be detrimental. Yet we must provide support to our staff who increasingly expect feedback, mentorship and reassurance. ${ }^{15}$

Speaking with colleagues is perceived to be a useful coping strategy ${ }^{816}$ and giving the participant control over how much to engage and disclose is thought to be important. ${ }^{16}$

With this in mind, Time Out has been developed in Leeds. Time Out is an adaptation of the Small Crisis Management Brief taken from Mitchell's Critical Incident Stress Management framework. ${ }^{17}$ The model has also been influenced by the Psychological First Aid approach. ${ }^{18}$

\section{TIME OUT PHILOSOPHY}

Time Out is a standardised method of providing support after any event that has the potential to cause distress. It can be requested by anyone. It is delivered by a clinical member of the team who has received facilitator training and is achievable in a busy unit, day or night. It works best delivered after an acute event and before the shift has ended. The meeting should last around $20 \mathrm{~min}$. 


\section{Box 1 Definition of debrief}

Verb-question (someone, typically a soldier or spy) about a completed mission or undertaking.

Noun-a series of questions about a completed mission or undertaking.

Synonym - question, quiz, interview, examine, crossexamine, interrogate, probe, sound out.

Antonym - answer, release, permit, sanction, let go, encourage, allow, help.

Definition of debrief, Oxford English Dictionary. https:// en.oxforddictionaries.com/definition/debrief (accessed 31 March 2019).

Synonyms and Antonyms for debrief. https://thesaurus.plus/ synonyms/debrief (accessed 31 March 2019).

The aim of Time Out is to provide an opportunity for colleagues to share their experiences in a safe and supportive environment and

- identify good practice,

- identify lessons learnt,

- identify any actions that need to be taken,

- promote peer support networks and

- signpost staff to other sources of support.

\section{TIME OUT-HOW IT WORKS}

There are four stages to the Time Out model (figure 2).

\section{Step 1: triggers}

Unexpected events of any nature are common triggers for a Time Out. Our triggers are set in very general terms to enable staff to have a low threshold in asking for a meeting:

- Cardiac or respiratory arrest or 2222 call.

- Any event which has the potential to cause distress to staff.

\section{Box 2 Terminology}

Acute stress disorder (also known as acute stress reaction)-applies in the first month after a traumatic event. It requires the presence of nine or more symptoms from any of the five categories of intrusion, negative mood, dissociation, avoidance and arousal. These can be starting or worsening after the traumatic event. ${ }^{10}$

Post-traumatic stress disorder-a range of symptoms associated with functional impairment, including re-experiencing, avoidance, hyperarousal (including hypervigilance, anger and irritability), negative alterations in mood and thinking, emotional numbing, dissociation, emotional dysregulation, interpersonal difficulties or problems in relationship, and negative self-perception. ${ }^{10}$ Moral distress - originally defined by Jameton (1984) as 'when one knows the right thing to do for a patient but institutional constraints make it impossible to do so'. Burnout - defined by Maslach (1996) as a triad of emotional exhaustion, depersonalisation and reduced personal accomplishment.

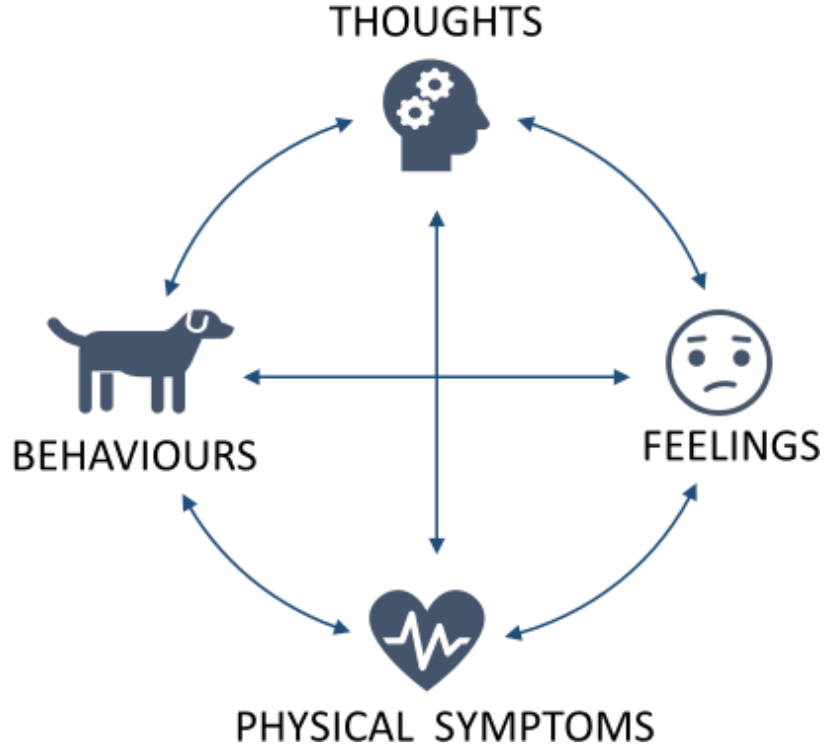

Figure 1 Relationship between thoughts, feelings, physical symptoms and behaviours.

You should identify potential triggers that apply in your area. Individual experiences are relevant; what might be stressful for someone inexperienced may not be for their experienced colleague and vice versa.

\section{Step 2: planning the meeting}

The only way of including everyone involved in the event is to hold the meeting during the same shift. Timing will depend on department workload and clinical commitments; buy-in is required from senior staff. Planning the meeting in advance helps to release staff from their clinical commitments to attend.

Everyone should be invited, including non-clinical staff who were involved in or witnessed the event, but nobody should be coerced into attending. Make it clear that attendance is entirely voluntary; it may be unhelpful for someone to attend if their preferred coping strategy is not to talk immediately afterwards with colleagues. Denial can be an adaptive short-term coping strategy; some may prefer to access informal support networks. ${ }^{14}$

The facilitator should

- involve the nurse in charge,

- collate a list of names and emails and invite all staff involved,

- arrange the time and location of the Time Out.

If it is not possible to hold the meeting on the same shift, then our practice is to offer a meeting within 48 hours, accepting that the opportunity to offer support to some members of the team will have been lost.

\section{Step 3: during the meeting}

Facilitating a Time Out meeting can be daunting for clinical staff. The facilitator may or may not have been involved in the event. Our facilitators use a prompt 


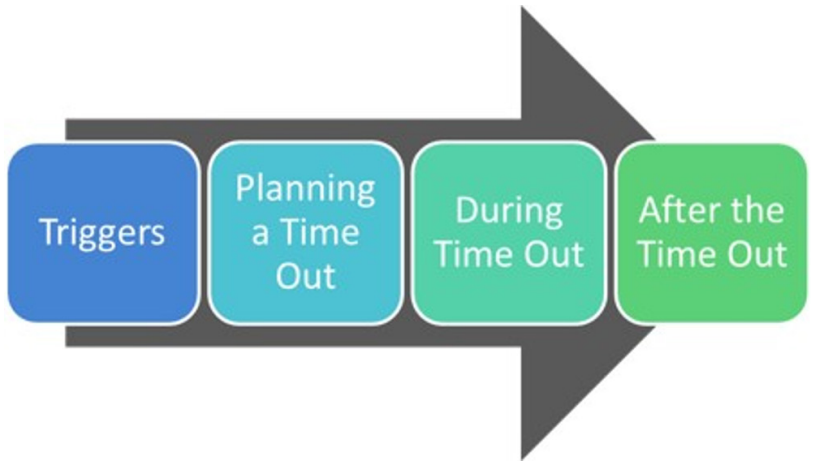

Figure 2 Four stages of the Time Out model.

when introducing the meeting to explain the aims of Time Out:

- Emphasise confidentiality and also that important lessons learnt will be disseminated.

- Reinforce that the purpose of the meeting is not about blame or investigation.

- Review events, gaining individual perspectives.

- Identify good practice.

- Identify lessons learnt and areas for development.

- Offer opportunity for all staff to speak and ask questions in a safe and supportive environment.

- Promote peer support.

- Signpost to other sources of support.

Notes are taken in the meeting, ideally by a second facilitator. Attendees complete a brief evaluation to give real time feedback to the facilitator on usefulness, what staff liked about the meeting and what they would change.

Further tips for facilitators are suggested in box 3 .

\section{Step 4: after the meeting}

The facilitator is responsible for ensuring that the following are completed:

- Write up the meeting-keep it anonymised and briefand email to all staff on the list (including those not attending) within 24 hours.

- Advise staff about further sources of support available locally (box 4).

- Give written information on common reactions (box 5) and tips on how to manage after a traumatic event (box 6).

- Disseminate learning points to the wider team as appropriate.

\section{Box 4 Examples of other sources of support}

Individual support from clinical supervisor/educational supervisor/line manager.

General practitioner.

Local Hospital Trust Occupational Health services/counselling services.

Local Improving Access to Psychological Therapies service.

Regional Deanery counselling service.

National Professional bodies and unions counselling

services.

\section{Box 3 Tips for facilitators}

Open question to start, for example, 'What would you like to talk about?'.

- Listen - giving your time and attention can be helpful.

- Validate their feelings.

- Be a compassionate colleague-do not judge, let staff know it is okay to be not okay.

- Offer comfort.

- You are giving protected time to stop, breathe, think and take stock.

Active listening

- Help the staff to verbalise the issues.

- Show you are listening by giving eye contact, nods of the head, open body language, sitting at the same level.

- Allow silence- this is part of showing you are listening and not just waiting for your turn to talk.

Empathise and reflect

- Reflect back what you think you have heard.

- Always try to be tentative just in case you have not understood correctly.

- Watch Brené Brown on Empathy by the RSA here in https://youtu.be/1 Evwgu369Jw.

Self-disclosure and sharing

- People can sometimes gain comfort when they hear if others have had a similar experience, but try to avoid saying, 'I know how you feel'.

Encourage discussion

- Explore experiences and encourage questions.

Strong feelings

- Try not to close down strong feelings and emotions.

- Avoid premature reassurance - instead of saying, 'Don't worry, you weren't to blame' instead try, 'What makes you think you are to blame?'.

Safety

- Keep the meeting safe, confidential and with clear boundaries.

- Challenge a colleague if they are being disrespectful, critical or blaming.

Ending

- Keep to time, allow final questions, summarise and praise.

Looking after yourself

- Remember that you are not expected to fix things.

- Peer support, being there and listening is valuable.

- Keep in touch with other facilitators and colleagues who have an interest in staff support.

- Delegate action points that need follow-up.

- Complete an incident form if appropriate.

- Notify local Time Out lead that a meeting has taken place.

- Collect evaluation forms and store in Time Out folder.

- Store confidential documents in a locked office or secure drive.

Maintain confidentiality by keeping attendance lists in a secure location and separate from the notes. No patient or staff should be named in the minutes, which only need reflect the themes discussed. If an individual is identified as needing further support, the facilitator should, with the permission of the staff 
Box 5 Common signs of a stress reaction following a traumatic event

\section{Physical}

Fatigue, nausea, muscle tremors, rapid heart rate, aches and pains, headaches, visual difficulties, dizziness, sweating, shock symptoms, chills.

\section{Cognitive}

Blaming someone, confusion, difficulty making decisions or concentrating, heightened alertness, poor problem-solving.

\section{Emotional}

Anxiety, guilt, grief, denial, fear, uncertainty, loss of emotional control, depression, feeling overwhelmed, intense anger, irritability, agitation, apprehension.

\section{Behavioural}

Withdrawal, isolation, emotional outbursts, suspiciousness, increased use of alcohol, inability to relax, startle response, pacing, difficulty sleeping, avoidance of situations/people/ objects.

member, refer to their clinical supervisor as would be usual practice.

\section{FACILITATORS}

To be able to offer this model of staff support consistently and equitably, it works best to train a network

\section{Box 6 Helpful hints for managing a traumatic}

event

\section{For yourself}

- Try to rest when you need to. Take time to process the incident.

- Speak with family and friends or people that you feel safe with.

- Remember recurring thoughts, dreams or intrusive visual images of the event are normal-they are your brain's way of trying to process the experience.

- Maintain as normal a schedule as possible.

- Eat well balanced and regular meals (even if you do not feel like it).

- Try to keep a reasonable level of activity.

- Physical exercise is often helpful.

- Express your feelings as they arise.

- If symptoms described (box 5) last more than 4 weeks or if they are particularly severe then seek help either via your general practitioner or your Hospital Trust staff support services, for example, staff counselling.

\section{For colleagues or friends}

- Offer your assistance and a listening ear.

- Spend time with the person.

- Reassure them they are safe.

- Help with everyday tasks that may have become difficult.

- Give them space when they need it.

- Don't take their anger or other feelings personally.

\section{Box 7 Facilitators-frequently asked questions}

Who can be a Time Out facilitator?

Any clinical member of the team-for example, a doctor, nurse or allied health professional-who is interested and enthusiastic about supporting their colleagues.

\section{What does facilitator training involve?}

In our hospital, we offer a 4-hour training session on the background to Time Out, the principles of promoting staff health and well-being, building resilience, recognising signs of burnout and PTSD and practical guidance on how to implement the model.

\section{Will I open up a can of worms by having a Time Out?}

It is possible that staff will become distressed during a Time Out. Staff tell us it can be of huge benefit to them to discuss any issues that arise in a safe and supportive environment and to allow each individual to express their viewpoint if they choose to. Facilitators need to be experienced enough to be able to maintain clear boundaries and to keep the meeting safe.

\section{What do I do if a serious issue arises between colleagues that cannot be resolved in the Time Out} meeting?

In our experience, this is rare. Facilitators need to be able to take control if needed and to state that the issue will need to be explored further and dealt with appropriately after the Time Out meeting.

\section{What support is available for facilitators?}

Each area should develop a Time Out team who can provide peer support for each other. We have set up a regular meeting for all Time Out facilitators in the hospital, supported by a psychologist, to enable staff to share and reflect on their experiences in implementing the model.

\section{Can I see an example of how Time Out actually works?}

Yes. You can watch a demonstration of the Time Out process here: https://youtu.be/rd7WLMKJY7U.

\section{I have never facilitated before. How do I get going? \\ The model works well using two facilitators. The less experienced facilitator will have support, and the main facilitator can focus on the group while the other takes notes. We have set up a network on a secure social media app to enable us to contact others to assist in running a meeting and create opportunities for new facilitators to become involved.}

of facilitators. We recommend a multiprofessional approach to this. See box 7 for a list of frequently asked questions.

\section{FOLLOW-UP}

We acknowledge that Time Out is just one part of our staff support structure, and that not all units have the benefit of psychological support. We recommend a 
Test your knowledge

1. Which three of the following apply to planning a Time Out meeting:

A. Unexpected events are common triggers.

B. A meeting should only be offered if senior staff think it is appropriate.

C. Only invite those who you think would want to attend.

D. Attendance is voluntary.

E. Planning a time and place involving the nurse in charge helps to release staff to attend.

2. The following three principles apply to a Time Out meeting:

A. The model is best delivered on the same shift with a duration of around 20 min.

B. The purpose of the meeting is to focus on clinical management only.

C. The meeting is confidential, but important lessons will be disseminated to the wider team.

D. All staff should be offered the opportunity to speak and ask questions.

E. It is assumed that staff will already know where to seek further support if needed.

3. Tips for facilitators include three of the following:

A. Start with a clinical summary of the case.

B. Show active listening by use of eye contact, nodding and open body language.

C. Explore experiences and encourage questions.

D. Try to reassure colleagues if they feel to blame.

E. Challenge a colleague if they are being disrespectful, critical or blaming.

4. The facilitator is responsible for ensuring which one of the following is completed:

A. Advise staff about further sources of support.

B. Give written information on common reactions and how to manage after a traumatic event.

C. Delegate any action points that need to be followed up.

D. Store confidential documents in a safe place.

E. All of the above.

5. Which three of the following are true following a traumatic event:

A. Recurring thoughts, dreams or intrusive visual images of the event are your brain's way of trying to process the experience.

B. Anxiety, guilt, grief, denial and fear are all common emotional responses.

C. Physical exercise should be avoided.

D. You should never tell anyone about your thoughts and feelings after a traumatic experience.

\section{Test your knowledge}

E. If symptoms last more than 4 weeks or if they are particularly severe then you should seek help and support via your general practitioner or staff counselling service.

Answers to the quiz are at the end of the references.

\section{SUCCESSES AND CHALLENGES}

Time Out meetings have been attended by staff from a wide range of disciplines and in varying locations. There are no objective data to show evidence of benefit or harm, or to measure quality and impact. Nevertheless, the model evaluates well by those attending, all of whom would recommend to a colleague, and 135 facilitators have been trained in our hospital. Themes from evaluations include observations on the supportive, safe and informal environment, openness and honesty from colleagues, educational value and ideas to improve clinical practice. Significant barriers include having enough facilitators to deliver the model equitably to all, raising awareness that support is available and changing mindsets.

\section{CONCLUSION}

Time Out is a standardised method of providing support to staff soon after a traumatic event. It can be delivered by clinical staff and offers some of the advantages of the clinical debrief while also promoting peer support, educating on common reactions and signposting to other sources of support. Building up a network of facilitators across professions and disciplines can support the process of embedding a culture of staff support in the workplace.

Acknowledgements The authors would like to thank Emor Miller, Nicola Lester and Santosh Sundararajan for their contribution to the development of the Time Out model. Thanks also to Andrea Brown who provided us with our tips for facilitators, in association with Leeds Teaching Hospitals Emergency Departments Listening Ears service, and to Jennie Ormerod for creating the written information we use on common reactions and tips on how to manage after a traumatic event.

Contributors SC wrote the first draft. MW contributed to the development of Time Out and helped with the literature review. JFE provided teaching materials for training facilitators which were used in the manuscript. MW and JFE reviewed and contributed to the final manuscript.

Funding The authors have not declared a specific grant for this research from any funding agency in the public, commercial or not-for-profit sectors.

Competing interests None declared.

Patient consent for publication Not required.

Provenance and peer review Commissioned; internally peer reviewed.

ORCID iDs

Sian Cooper http://orcid.org/0000-0002-9242-5078

Mark Winton http://orcid.org/0000-0003-4303-9602

\section{REFERENCES}

1 Patient Safety network, U.S. Department of Health and Human Services. Debriefing for clinical learning. Available: https:// 
psnet.ahrq.gov/primers/primer/36/debriefing-for-clinicallearning [Accessed 31 Mar 2019].

2 Zigmont JJ, Kappus LJ, Sudikoff SN. The 3D model of Debriefing: defusing, discovering, and deepening. Semin Perinatol 2011;35:52-8.

3 May N. It's Good to Talk - Debrief in the Emergency Department. St Emlyn's, 2013. Available: http://www. stemlynsblog.org/good-to-talk-debrief-in-the-emergencydepartment/ [Accessed 31 Mar 2019].

4 Carley S. Five free strategies to improve your resuscitation practice. St Emlyn's, 2018. Available: http://www.stemlynsblog. org/stemlynslive-five-free-strategies-to-improve-yourresuscitation-practice-st-emlyns/ [Accessed 31 Mar 2019].

5 Davis M, Denning K. Listening through the learning conversation: a thought provoking intervention. MedEdPublish 2018;7:61.

6 Ireland S, Gilchrist J, Maconochie I. Debriefing after failed paediatric resuscitation: a survey of current UK practice. Emerg Med J 2008;25:328-30.

7 Hollingsworth CE, Wesley C, Huckridge J, et al. Impact of child death on paediatric trainees. Arch Dis Child 2018;103:14-18.

8 Colville GA, Smith JG, Brierley J, et al. Coping with staff burnout and work-related posttraumatic stress in intensive care. Pediatr Crit Care Med 2017;18:e267-73.

9 Rose S, Bisson J, Churchill R, et al. Psychological Debriefing for preventing post traumatic stress disorder (PTSD). Cochrane Database Syst Rev 2002:CD000560.

10 Post-traumatic stress disorder. NICE guideline [NG116], 2018. Available: https://www.nice.org.uk/guidance/ng116 [Accessed 31 Mar 2019].

11 Prentice T, Janvier A, Gillam L, et al. Moral distress within neonatal and paediatric intensive care units: a systematic review. Arch Dis Child 2016;101:701-8.

12 Jones GAL, Colville GA, Ramnarayan P, et al. Psychological impact of working in paediatric intensive care. A UK-wide prevalence study. Arch Dis Child 2020;105:470-5.

13 NHS Staff and Learner's Mental Wellbeing Commission. Health education England, February, 2019. Available: https:// www.hee.nhs.uk/sites/default/files/documents/NHS\%20\% 28HEE\%29\%20-\%20Mental\%20Wellbeing\%20Commission\% 20Report.pdf [Accessed 31 Mar 2019].
14 Ormerod J. Current research into the effectiveness of Debriefing. psychological Debriefing: professional practice board Working Party (Chapter 2). The British Psychological Society, 2002.

15 Jones K, Warren A, Davies A. Mind the gap. exploring the needs of early career nurses and midwives in the workplace. summary report from Birmingham and Solihull local education and training Council every student counts project. published may, 2015. Available: https://www.nhsemployers.org/-/media/ Employers/Documents/Plan/Mind-the-Gap-Smaller.pdf [Accessed 31 Mar 2019].

16 Devilly GJ, Cotton P. Psychological Debriefing and the workplace: defining a concept, controversies and guidelines for intervention. Aust Psychol 2003;38:144-50.

17 Mitchell JT, Everly GS. Psychological Debriefing; theory, practice and evidence. Chapter 5: critical incident stress debriefings; evolutions, effects and outcomes. Cambridge University Press, 2000.

18 Freeman C, Flitcroft A, Weeple P. Psychological first aid: a replacement for psychological Debriefing. Short-term post trauma responses for individuals and groups. The CullenRivers Centre for Traumatic Stress, Royal Edinburgh Hospital, 2003.

19 Brewin CR, Rose S, Andrews B, et al. Brief screening instrument for post-traumatic stress disorder. Br J Psychiatry 2002;181:158-62.

20 Walters JTR, Bisson JI, Shepherd JP. Predicting posttraumatic stress disorder: validation of the trauma screening questionnaire in victims of assault. Psychol Med 2007;37:14350 .

\section{Answers to the multiple choice questions}

1. $A, D, E$.

2. $A, C, D$.

3. $B, C, E$.

4. $E$.

5. $A, B, E$. 